\title{
Simultaneous Material and Topology Optimization Based on Topological Derivatives
}

\author{
Jannis Greifenstein $^{(\varpi)}$ and Michael Stingl \\ Chair of Applied Mathematics 2, \\ Friedrich-Alexander-Universität Erlangen-Nürnberg (FAU), \\ Nägelsbachstr. 49b, 91052 Erlangen, Germany \\ \{greifenstein, stingl\}@math.fau.de \\ http://www.math.fau.de
}

\begin{abstract}
We use an asymptotic expansion of the compliance cost functional in linear elasticity to find the optimal material inside elliptic inclusions. We extend the proposed method to material optimization on the whole domain and compare the global quality of the solutions for different inclusion sizes. Specifically, we use an adjusted free material optimization problem, that can be solved globally, as a global lower material optimization bound. Finally, the asymptotic expansion is used as a topological derivative in a simultaneous material and topology optimization problem.
\end{abstract}

Keywords: Material optimization - Topology optimization - Material orientation - Asymptotic expansions · Discrete material optimization

\section{Introduction}

We investigate material and topology optimization of compliance problems in two dimensions. To this end, we first present an asymptotic formula ${ }^{1}$ of the compliance functional for the insertion of a number of ellipsoidal bodies in an elastic domain, originally derived in [1]. Later, we study numerically the feasibility of replacing all material using the same asymptotic expansion as for the ellipses and finally make use of the formula as topological derivative.

The problem described above is by far not new. There are many publications dealing with similar types of problems. For the rotational optimization considered later, in [2] an analytical formula for the strain energy is derived, to directly compute the optimal material orientation. In [3], this has been embedded into a structural optimization algorithm for compliance minimization. A similar approach is discussed in [4] for a plate model. The method proposed in this article, however, can be used for a broader spectrum in material optimization as well,

The authors want to thank the German Research Foundation (DFG) for funding this research work within Collaborative Research Centre 814, subproject C2.

${ }^{1}$ We note that the asymptotic formulae are also available for the three-dimensional case.

(C) IFIP International Federation for Information Processing 2014

C. Pötzsche et al. (Eds.): CSMO 2013, IFIP AICT 443, pp. 118-127, 2014.

DOI: $10.1007 / 978-3-662-45504-3 \_11$ 
such as discrete material optimization. The algorithm for simultaneous material and topology optimization presented at the end of this article is very similar to topology gradient methods, see e.g. [5]. For more references, see [6].

\section{Material and Topology Optimization}

We consider a domain $\Omega \subset \mathbb{R}^{2}$ of isotropic elastic material. The domain is subject to exterior traction and other boundary conditions (e.g. homogeneous Dirichlet conditions). The objective is to find the optimal material $C^{0}$ in a set of admissible materials $\mathcal{C}$ to insert into an inclusion, for which the compliance as defined in (1) is minimized.

The elastic body is modeled by the equation of linear elasticity

$$
\int_{\Omega} C_{i j k l}(x) \varepsilon_{i j}(u) \varepsilon_{k l}(v) \mathrm{dx}=\int_{\Gamma} f u \mathrm{ds},
$$

with the displacement field $u$, the linearized strains $\varepsilon_{i j}(u)=\left(\frac{\partial u_{i}}{\partial x_{j}}+\frac{\partial u_{j}}{\partial u_{i}}\right)$ and a traction $f$. We use Voigt notation to denote the fourth-order stiffness tensor $C_{i j k l}$ by a symmetric $3 \times 3$-matrix and the strains and stresses by a vector

$$
C=\left(\begin{array}{ccc}
C_{1111} & C_{1122} & \sqrt{2} C_{1112} \\
& C_{2222} & \sqrt{2} C_{2212} \\
\text { sym. } & 2 C_{1212}
\end{array}\right), \quad \varepsilon=\left(\begin{array}{c}
\varepsilon_{11} \\
\varepsilon_{22} \\
\sqrt{2} \varepsilon_{12}
\end{array}\right), \quad \sigma=\left(\begin{array}{c}
\sigma_{11} \\
\sigma_{22} \\
\sqrt{2} \sigma_{12}
\end{array}\right) .
$$

The stresses are given by Hooke's law

$$
\sigma=C \varepsilon
$$

\subsection{Optimal Material in Elliptic Inclusions}

We insert a finite number of inclusions $\omega_{i}, i=1, \ldots, n_{\text {ell }}$ with $n_{\text {ell }}>1$ and centers $z_{1}, \ldots, z_{n_{\text {ell }}}$ into the domain $\Omega$ and search for the optimal material to be used in the inclusions. For an exemplary setup of boundary conditions and loads, a sketch of a possible problem specification is shown in Fig. 1. We place the elliptic inclusions on a regular grid within the domain $\Omega$, so that the center points of the inclusions are distributed equidistantly. In the sketch in Fig. 1, we have $n_{\mathrm{ell}}=100$ disjoint elliptic inclusions. In the remaining domain

$$
\Omega^{1}:=\Omega \backslash \bigcup_{i=1}^{n_{\mathrm{ell}}} \omega_{i}
$$

we insert an isotropic matrix material $C^{1}$, and, in each inclusion, a material $C_{i}$, $i=1, \ldots, n_{\mathrm{ell}}$. 


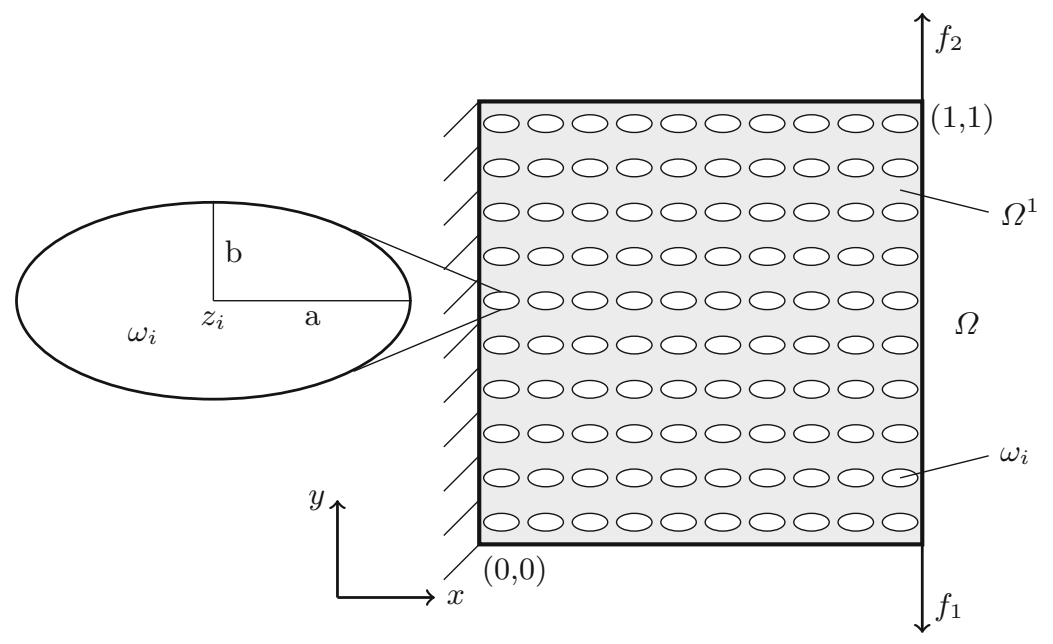

Fig. 1. Example for placing elliptic inclusions $\omega_{i}, i=1, \ldots, n_{\mathrm{ell}}$, in the domain $\Omega$.

Now, the compliance function of the elastic body $\Omega$ (without inclusions) with respect to a set of given load functions $f_{k} \in\left[L^{2}(\Gamma)\right]^{2}, k \in \mathcal{K}=\left\{1,2, \ldots, n_{\text {loads }}\right\}$ applied to a part $\Gamma$ of $\partial \Omega$ is defined as

$$
\mathcal{J}_{c}(f)=\sum_{k \in \mathcal{K}} \int_{\Gamma} f_{k}(x) u_{k}(x) \mathrm{ds} .
$$

By virtue of the asymptotic expansion (cf. [1]) for the two-dimensional case, the compliance function $\mathcal{J}_{c\left(C^{0}\right)}(f)$ of a body with a single inclusion of material $C^{0}$ can be approximated as

$$
\left|\mathcal{J}_{c\left(C^{0}\right)}(f)-\left(\mathcal{J}_{c}(f)-\frac{h^{2}}{2} \varepsilon(u ; z)^{\top} P_{\left(C^{0}\right)} \varepsilon(u ; z)\right)\right| \leq c h^{5 / 2}(1+|\ln h|)\|f\|^{2},
$$

where $h$ is a dimensionless scaling parameter for the elliptic inclusion $\omega, \varepsilon(u ; z)$ is the strain corresponding to the displacement of the domain $\Omega$ without inclusion evaluated at the center $z$ of the ellipse, and $P_{\left(C^{0}\right)}$ is the so called polarization matrix given by

$$
P_{\left(C^{0}\right)}=-|\omega|\left(C^{1}-C^{0}+\left(C^{1}-C^{0}\right)\left(\mathbb{I}_{3}-\Psi\left(C^{1}-C^{0}\right)\right)^{-1} \Psi\left(C^{1}-C^{0}\right)\right) .
$$

The matrix $\Psi$ in formula (3) depends solely on the isotropic matrix material and is, for the stretched coordinates $\omega=\left\{\xi: \xi_{1}^{2} / a^{2}+\xi_{2}^{2} / b^{2}<1\right\}, \xi=x / h$, computed by

$$
\Psi=\int_{\partial \omega} D(\nu(\xi))\left(D\left(\nabla_{\xi}\right) \Phi(\xi)\right)^{\top} \mathrm{ds}_{\xi}, \quad D(x)^{\top}=\left(\begin{array}{ccc}
x_{1} & 0 & 2^{-1 / 2} x_{2} \\
0 & x_{2} & 2^{-1 / 2} x_{1}
\end{array}\right),
$$


where $\nu$ denotes the exterior normal of the ellipse $\omega$ and $a, b$ are the semi-major and semi-minor axes of the ellipse, respectively, cf. Fig. 1. Finally, $\Phi$ in (4) is the fundamental solution given by

$$
\Phi(x)=c_{1}\left(\begin{array}{cc}
-(\lambda+3 \mu) \ln (r)-(\lambda+\mu) \frac{x_{2}^{2}}{r^{2}} & (\lambda+\mu) \frac{x_{1} x_{2}}{r^{2}} \\
(\lambda+\mu) \frac{x_{1} x_{2}}{r^{2}} & -(\lambda+3 \mu) \ln (r)-(\lambda+\mu) \frac{x_{1}^{2}}{r^{2}}
\end{array}\right),
$$

where $c_{1}=\frac{1}{4 \pi} \frac{1}{\mu(\lambda+2 \mu)}, r=\sqrt{x_{1}^{2}+x_{2}^{2}}$ and $\lambda$ and $\mu$ are the Lamé parameters corresponding to the isotropic material $C^{1}$, see e.g. [7, Chap. 3]. A more detailed explanation may be found in [1, Sect. 3.1].

In order to minimize the compliance for a single inclusion with center $z_{i}$ we can now use the asymptotic expansion (2) and The inserted material will be chosen out of a set of admissible materials $\mathcal{C}$

$$
\begin{aligned}
\min _{C^{0} \in \mathcal{C}} \mathcal{J}_{c\left(C^{0}\right)}(f) & \approx \min _{C^{0} \in \mathcal{C}} \mathcal{J}_{c}(f)-\frac{h^{2}}{2} \varepsilon\left(u ; z_{i}\right)^{\top} P_{\left(C^{0}\right)} \varepsilon\left(u ; z_{i}\right) \\
& =\mathcal{J}_{c}(f)+\frac{h^{2}}{2} \min _{C^{0} \in \mathcal{C}}\left(-\varepsilon\left(u ; z_{i}\right)^{\top} P_{\left(C^{0}\right)} \varepsilon\left(u ; z_{i}\right)\right) .
\end{aligned}
$$

Thus, taking into account that $J_{c}(f)$ is independent of the rotation angle and noting that $h^{2}$ is a constant scaling parameter, the functional

$$
\mathcal{D}_{c}\left(C^{0}, z_{i}\right):=-\varepsilon\left(u ; z_{i}\right)^{\top} P_{\left(C^{0}\right)} \varepsilon\left(u ; z_{i}\right)
$$

can be used as an approximate model to find the optimal rotation angle resulting in the optimization problem

$$
\min _{C^{0} \in \mathcal{C}} \mathcal{D}_{c}\left(C^{0}, z_{i}\right)
$$

We note that the functional $\mathcal{D}_{c}$ depends on the inserted material $C^{0}$ only via the polarization matrix $P_{\left(C^{0}\right)}$ and on the displacement field $u(x)$ only locally through the evaluation of the strain at center $z_{i}$ of the ellipse. Furthermore, only a single evaluation of the state problem for the unperturbed domain is required.

Due to the local character of (6) the optimal orientation of $n_{\text {ell }}<\infty$ nonintersecting inclusions at once can be approximated by the solution of $n_{\text {ell }}$ optimization problems of type (7) or equivalently by the solution of the problem

$$
\min _{\left(C_{0}^{0}, \ldots, C_{n_{\mathrm{ell}}}^{0}\right) \in \mathcal{C}^{n_{\mathrm{ell}}}} \mathcal{J}_{c\left(C_{0}^{0}, \ldots, C_{n_{\mathrm{ell}}}^{0}\right)}^{\text {asymp }}:=\sum_{i=1}^{n_{\mathrm{ell}}} \min _{C_{i}^{0}} D_{c}\left(C_{i}^{0}, z_{i}\right)
$$

which is separable in terms of the different materials $C_{0}^{0}, \ldots, C_{n_{\text {ell }}}^{0}$ used in the different inclusions. The latter is certainly only an approximation of the original simultaneous material optimization problem

$$
\min _{\left(C_{0}^{0}, \ldots, C_{n_{\mathrm{ell}}}^{0}\right) \in \mathcal{C}^{n_{\mathrm{ell}}}} \mathcal{J}_{c\left(C_{0}^{0}, \ldots, C_{n_{\mathrm{ell}}}^{0}\right)},
$$


however it is shown in [6] for the optimal rotation of an orthotropic material, that the results for this approximation are close to the solution of the original problem. Moreover, we will later on compare the quality of the approximated solution to rigorous lower bounds. We want to stress that still only a single evaluation of the state problem for the unperturbed domain is required, which allows for a highly efficient numerical solution.

The optimization procedure for solving (8) is performed by the following algorithm:

(S1) choose matrix material $C^{1}$ and admissible materials for inclusions $\mathcal{C}$;

(S2) compute $\Psi$ from (4);

(S3) define loads and boundary conditions;

(S4) solve state problem without inclusions for isotropic material;

foreach inclusion $\omega_{i}, i=1, \ldots, n_{\text {ell }}$ do

I (S5) solve $\min _{C_{i}^{0} \in \mathcal{C}} \mathcal{D}_{c}\left(C_{i}^{0}, z_{i}\right)$ to global optimality;

end

Algorithm 1. Basic algorithm for minimization of the compliance based on the asymptotic model.

\subsection{Admissible Material Choices}

In order to avoid local minima, the set of admissible materials $\mathcal{C}$ should allow for a global solution of the local material optimization problem (7) in a reasonable time. Interestingly, the easiest choice here would be a set of discrete materials. In the following, however, we concentrate on parametric material formulations. Using the properties of the asymptotic expansion, we can give a wider class of parametrizations that lead to globally solvable problems. According to [6] (Theorem 2.8, p. 14), the polarization matrix (3) is positive definite if $\left(C^{0}\right)^{-1}-$ $\left(C^{1}\right)^{-1}$ is negative definite. If the isotropic material $C^{1}$ is then chosen s.t. every $C^{0} \in \mathcal{C}$ is strictly stiffer, it can then be shown that the functional (6) is convex for any linear material parametrization. An example of this would be the so-called free material optimization (FMO, see e.g. $[8,9])$ :

$$
\mathcal{C}_{\mathrm{FMO}}:=\left\{\left(\begin{array}{lll}
e_{1} & e_{2} & e_{3} \\
e_{2} & e_{4} & e_{5} \\
e_{3} & e_{5} & e_{6}
\end{array}\right) \succeq \underline{\tau}\left(\begin{array}{lll}
1 & 0 & 0 \\
0 & 1 & 0 \\
0 & 0 & 1
\end{array}\right), e_{1}+e_{4}+e_{6}=\bar{\rho}\right\},
$$

where $\underline{\tau}$ is a fixed lower eigenvalue bound and $\bar{\rho}$ bounds the total stiffness of the material tensor. The positivity constraint is still linear as a semidefinite program, s.t. the resulting problem stays convex.

Furthermore, we will now consider the usually difficult problem of rotational optimization that is also vastly simplified by the breakdown to local minimization problems. An example of this would be the variation of angle and stiffness

$$
C^{0}(\theta, s):=\Theta(\theta)^{\top} C(s) \Theta(\theta), C(s):=\left(\begin{array}{ccc}
1.0+s & 0.5 & 0 \\
0.5 & 15-s & 0 \\
0 & 0 & 1.0
\end{array}\right)
$$


where we rotate an orthotropic material by an angle $\theta$ using the orthogonal rotation matrix $\Theta$ defined as

$$
\Theta(\theta)=\left(\begin{array}{ccc}
\cos (\theta)^{2} & \sin (\theta)^{2} & -\sqrt{2} / 2 \sin (2 \theta) \\
\sin (\theta)^{2} & \cos (\theta)^{2} & \sqrt{2} / 2 \sin (2 \theta) \\
\sqrt{2} / 2 \sin (2 \theta) & -\sqrt{2} / 2 \sin (2 \theta) & \cos (2 \theta)
\end{array}\right) .
$$

In the results section, we will consider two different admissible material sets based on this parametrization, namely

$$
\mathcal{C}_{\theta, s}:=\left\{C^{0}(\theta, s): \theta \in[0, \pi], s \in[0,14]\right\}
$$

and a pure rotational optimization

$$
\mathcal{C}_{\theta, s=0}:=\left\{C^{0}(\theta, s): \theta \in[0,2 \pi], s=0\right\} .
$$

Now this parametrization fails the proposed linearity. However, for any fixed rotation angle $\theta$, the local minimization problems (8) are still strictly convex. It follows, that the problem can be solved as a bilevel problem

$$
\begin{gathered}
\min _{\theta_{i}, s_{i}} \mathcal{J}_{c}\left(C_{i}^{0}\left(\theta_{i}, s_{i}\right)\right)=\min _{\theta_{i}} G\left(\theta_{i}\right) \\
G\left(\theta_{i}\right):=\min _{s_{i}} \mathcal{J}_{c\left(C^{0}\left(\theta_{i}, s_{i}\right)\right)},
\end{gathered}
$$

which still allows for a global solution with moderate cost as there is only a single primary variable left. Note that this could be done similarly for more complicated linear parametrizations with rotation or in $3 \mathrm{D}$ with $2-3$ rotation angles.

Lastly, we consider an orthotropic material parametrization using engineering constants with fixed Poisson's ratio $\nu_{x y}=\nu_{y x}=0.3$ :

$$
C\left(\theta, E_{x}, E_{y}, G_{x y}\right)=\Theta(\theta)^{\top}\left(\begin{array}{ccc}
\frac{E_{x}}{1-0.09} & \frac{\sqrt{0.09 E_{x} E_{y}}}{1-0.09} & 0 \\
\frac{\sqrt{0.09 E_{x} E_{y}}}{1-0.09} & \frac{E_{y}}{1-0.09} & 0 \\
0 & 0 & 2 G_{x y}
\end{array}\right) \Theta(\theta),
$$

with elasticity moduli $E_{x}, E_{y}$, shear modulus $G_{x y}$ and $\Theta$ as in (12). We define the admissible material set corresponding to (13) as

$$
\mathcal{C}_{\text {Eng }}:=\left\{C\left(\theta, E_{x}, E_{y}, G_{x y}\right): \theta \in[0, \pi], E_{x}, E_{y} \in[1,15], G_{x y} \in[0.5,7.5]\right\} .
$$

\subsection{From Elliptic Inclusions to Material Optimization}

While the asymptotic model (2) rigorously holds only for elliptic inclusions of small size, in the following we will also numerically investigate the behavior when replacing the material inside squared patches of finite elements. Choosing the elements properly, the material in the whole domain can be replaced this way with the FE patches still being disjoint. Using a large number of patches, the size of the inclusion stays small compared to the domain size. Thus, we will study increasingly bigger ellipses and compare the compliance values of the different parametrizations to global lower material optimization bounds computed with an FMO solver. 
Validation Methods. For the numerical evaluation, we discretize the domain $\Omega$ using rectangular finite elements. This discretization is necessary to compute the displacements used in the asymptotic expansion. The elliptic inclusions are approximated by those finite elements, for which the coordinates of their center point are contained in the inclusion $\omega_{i}$. In order to obtain the actual compliance value for the optimization result, the material used in those elements is then replaced by the optimal value of $C_{i}^{0}$. When replacing all material, we use equally sized squared FE patches that are uniformly distributed, disjoint and cover the whole domain.

Furthermore, we compare the compliance values to a global lower material optimization bound determined by solving a modified FMO problem. Specifically, we solve

$$
\min _{\left(C_{0}^{0}, \ldots, C_{n_{\mathrm{ell}}}^{0}\right) \in \mathcal{C}_{\mathrm{FMO}}^{n_{\mathrm{ell}}}} \mathcal{J}_{c\left(C_{0}^{0}, \ldots, C_{n_{\mathrm{ell}}}^{0}\right)}
$$

with $\mathcal{C}_{\mathrm{FMO}}$ as in (10) and the bounds $\underline{\tau}$ and $\bar{\rho}$ chosen as close as possible to the ones used in the specific material parametrization, s.t. all possible tensors are a subset of $\mathcal{C}_{\mathrm{FMO}}$. For a more detailed description of the method, see [6]. Note that within these bounds, any physically admissible material may be used and that this problem is convex for the compliance cost functional. Thus, the problem is solved globally using the algorithm described in [9] and we obtain a global lower compliance bound.

Numerical Results. We consider the example from Fig. 1 with $10 \times 10$ ellipses and discretize the domain $\Omega$ using $100 \times 100$ finite elements. We compare the different admissible material sets as defined in Sect.2.2. For $\mathcal{C}_{\text {FMO }}$ we choose $\underline{\tau}=1$ as lower eigenvalue bound and $\bar{\rho}=17$ as upper trace bound both in the asymptotic material optimization as in the FMO solver. The results are shown in Table 1 and a visualization in Fig. 2. Although the error compared to the exact FMO result increases heavily with the size of the inclusions, this is largely due to the decrease of the overall compliance value. The absolute value does not increase much from the largest ellipses to the squared FE patches.

For the squared FE patches, we furthermore study the different parametrizations separating the domain into $50 \times 50$ patches. The results are found in Table 2 and Fig. 3(a). We can see, that for the parametrization with nonlinear

Table 1. Compliance values and FMO comparison for increasing ellipse size.

\begin{tabular}{l|l|l|l|l|l|l|l|r}
\hline$a=b:$ & 0.02 & & 0.04 & & 0.05 & & \multicolumn{2}{|c}{ squared patch } \\
\hline $\mathcal{C}_{\theta, s=0}$ & 15.420 & $1.0 \%$ & 11.053 & $6.15 \%$ & 8.3173 & $14.2 \%$ & 4.3730 & $42.3 \%$ \\
\hline $\mathcal{C}_{\theta, s}$ & 15.332 & $0.42 \%$ & 10.738 & $3.12 \%$ & 7.7140 & $5.93 \%$ & 3.5668 & $16.1 \%$ \\
\hline $\mathcal{C}_{\text {Eng }}$ & 15.328 & $0.39 \%$ & 10.647 & $2.25 \%$ & 7.6135 & $4.55 \%$ & 3.5014 & $13.9 \%$ \\
\hline $\mathcal{C}_{\text {FMO }}$ & 15.289 & $0.14 \%$ & 10.551 & $1.33 \%$ & 7.4840 & $2.77 \%$ & 3.2912 & $7.10 \%$ \\
\hline FMO & 15.268 & & 10.413 & & 7.2821 & & 3.0730 & \\
\hline
\end{tabular}




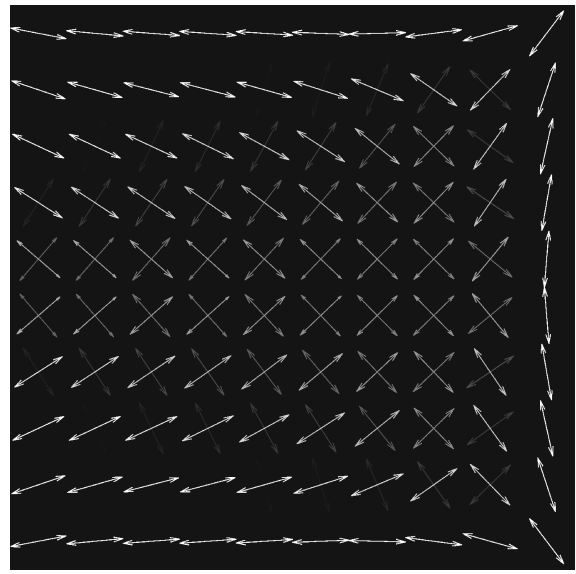

(a) $a=b=0.02$

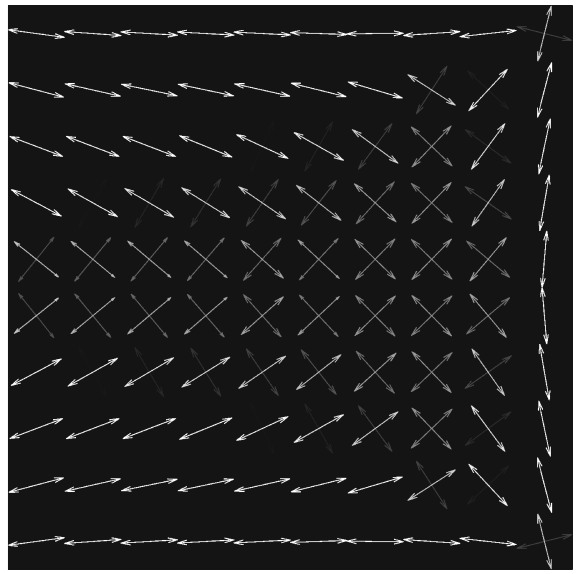

(b) square

Fig. 2. Optimization for $\mathcal{C}_{\theta, s}$ with similar results. The arrows denote the principal stiffness directions and the gray value the magnitude (black: low, white: high).

Table 2. Compliance values and FMO comparison for 50x50 squared FE patches.

\begin{tabular}{l|r|r}
\hline $\mathcal{C}_{\theta, s=0}$ & 3.6850 & $38.89 \%$ \\
\hline $\mathcal{C}_{\theta, s}$ & 2.9963 & $12.93 \%$ \\
\hline $\mathcal{C}_{\text {Eng }}$ & 3.0535 & $15.08 \%$ \\
\hline $\mathcal{C}_{\text {FMO }}$ & 2.8964 & $9.16 \%$ \\
\hline FMO & 2.6533 & \\
\hline
\end{tabular}

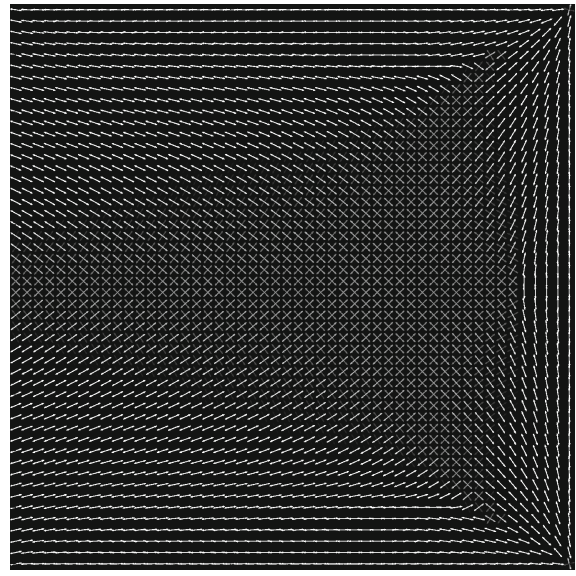

(a) square

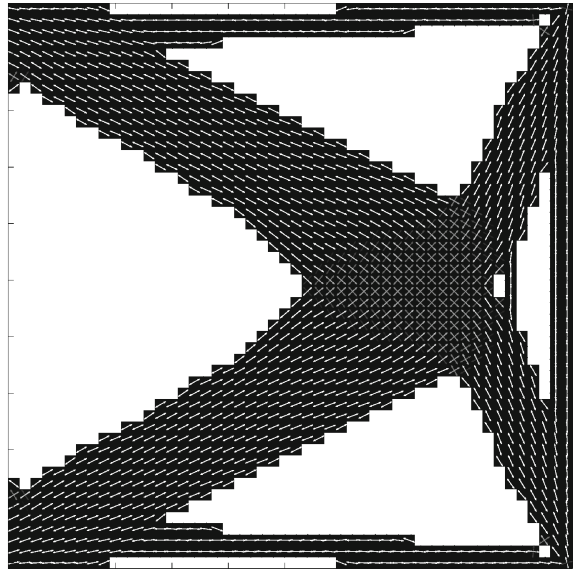

(b) square, topology

Fig. 3. Results of the simultaneous material and topology optimization for $\mathcal{C}_{\theta, s}$. 
subproblems $\mathcal{C}_{\text {Eng }}$, apparently a local minimum was found, which stresses the importance of a global solution of the local material optimization problems.

\subsection{Material and Topology Optimization}

The term (6) in the asymptotic expansion actually corresponds to a topological derivative and can hence be used for topology optimization. We propose an algorithm, where we do not only use the information of "drilling a hole", but use the values obtained in material optimization instead. The idea is, that those finite element patches, which provide the smallest gain, when substituting the optimal material $C^{0}$, are left out in the following iteration:

(S1) choose matrix material $C^{1}$ and admissible materials for inclusions $\mathcal{C}$;

(S2) compute $\Psi$ from (4);

(S3) define loads and boundary conditions;

while volume $>$ bound do

(S4) solve state problem without inclusions for current topology;

foreach inclusion $\omega_{i}, i=1, \ldots, n_{\text {ell }}$ do

| (S5) solve $\min _{C_{i}^{0} \in \mathcal{C}} \mathcal{D}_{c}\left(C_{i}^{0}, z_{i}\right)$ to global optimality;

end

(S6) remove FE-patches with $\min _{C_{i}^{0} \in \mathcal{C}} \mathcal{D}_{c}\left(C_{i}^{0}, z_{i}\right)$ largest;

\section{end}

Algorithm 2. Basic algorithm for simultaneous material and topology optimization based on the asymptotic model.

Note that, again, in each iteration the state problem only needs to be solved once.

In Fig. 3(b), the result of the algorithm for the admissible material set $\mathcal{C}_{\theta, s}$ and $50 \times 50$ squared $\mathrm{FE}$ patches is shown. In this experiment, we removed in the iteration $k$ a total of $200 * 0.83^{k} \mathrm{FE}$ patches until $3 \mathrm{FE}$ patches or less where removed, which lead to a final volume fraction of 0.542 . The strategy for the removal of ellipses can be varied, however a decreasing volume fraction should be removed in order to obtain a smoother convergence.

\section{Conclusion}

We proposed an efficient algorithm for material optimization on multiple elliptic inclusions. The numerical evidence suggests that the accuracy of the proposed method decreases only slightly, when replacing all material instead of just the material in elliptic inclusions. A major advantage of this algorithm is the possibility of avoiding local minima, however at the cost of only having an approximate solution. The total error in the studied example for the finer resolution was about $10 \%$. From experience in practice, the proposed algorithm for simultaneous material and topology optimization seems to work well in large parts, however oftentimes small bars are left over and if a hole happens to be drilled in a "bad" position the algorithm struggles, as material is not reintroduced. 
Nevertheless, both algorithms allow for a very efficient solution of usually quite complicated problems, such as discrete material optimization and rotational optimization. When the accuracy provided in this method does not suffice or the optimized topology appears flawed, the optimization result may still be used as a high quality initial design for other solution schemes, such as fully parametrized approaches.

\section{References}

1. Leugering, G., Nazarov, S., Schury, F., Stingl, M.: The Eshelby theorem and application to the optimization of an elastic patch. SIAM J. Appl. Math. 72(2), 512-534 (2012)

2. Pedersen, P.: On optimal orientation of orthotropic materials. Struct. Multidiscip. Opt. 1, 101-106 (1989)

3. Pedersen, P.: On thickness and orientational design with orthotropic materials. Struct. Multidiscip. Opt. 3, 69-78 (1991)

4. Thomsen, J.: Optimization of composite discs. Struct. Multidiscip. Opt. 3, 89-98 (1991)

5. Lewiński, T., Sokołowski, J.: Energy change due to the appearance of cavities in elastic solids. Int. J. Solids Struct. 40(7), 1765-1803 (2003)

6. Schury, F., Greifenstein, J., Leugering, G., Stingl, M.: On the efficient solution of a patch problem with multiple elliptic inclusions. Optim. Eng. (Accepted for publication, 2014)

7. Love, A.E.H.: A Treatise on the Mathematical Theory of Elasticity. Dover Publications, New York (1944)

8. Haslinger, J., Kočvara, M., Leugering, G., Stingl, M.: Multidisciplinary free material optimization. SIAM J. Appl. Math. 70(7), 2709-2728 (2010)

9. Stingl, M., Kočvara, M., Leugering, G.: A sequential convex semidefinite programming algorithm with an application to multiple-load free material optimization. SIAM J. Opt. 20(1), 130-155 (2009) 